\title{
A study to develop a robust method for measuring the detection efficiency of free-running InGaAs/InP single-photon detectors
}

\author{
M. López ${ }^{1 *}$ (D, A. Meda², G. Porrovecchio ${ }^{3}$, R.A. Starkwood (Kirkwood) ${ }^{4}$, M. Genovese², G. Brida² ${ }^{2}$ M. Šmid ${ }^{3}$, \\ C.J. Chunnilall ${ }^{4}$, I.P. Degiovanni ${ }^{2}$ and S. Kück
}

\author{
*Correspondence: \\ marco.lopez@ptb.de \\ 1Physikalisch-Technische \\ Bundesanstalt (PTB), Bundesallee \\ 100, 38116 Braunschweig, Germany \\ Full list of author information is \\ available at the end of the article
}

\begin{abstract}
The challenges faced in a comparison of measuring the detection efficiency of free-running InGaAs/InP single-photon avalanche detectors (InGaAs/InP SPAD) were studied by four European National Metrology Institutes (NMIs) meeting at a single laboratory. The main purpose of this study is to develop a trustable measurement technique and to provide a snapshot of the methods used by the four NMIs for measuring such photon-counting detectors at telecom wavelengths in order to establish proper procedures for characterising such devices. The detection efficiency measurements were performed using different experimental setups and reference standards with independent traceability chains at the wavelength of $1550 \mathrm{~nm}$. A dedicated model to correct the dead time and dark count effects on the SPAD's free-running counting process was developed, allowing the correct value of the photon rate impinging on the detector to be recovered from simple ratemeter measurements. The detection efficiency was measured for mean photon number per pulse between 0.01 and 2.4, corresponding to photon rates between approximately 1100 photon/s and 193,000 photon/s, respectively. We found that the measured values reported by the participants are all consistent within the stated uncertainties, proving the consistency of the measurement approach developed.
\end{abstract}

Keywords: Quantum technology; Quantum radiometry; Detection efficiency; Single-photon detectors; Single-photon sources; Metrology

\section{Introduction}

InGaAs/InP single-photon avalanche detectors (SPADs) operated in Geiger mode are today the most frequently used detectors in practical single-photon-based applications such as quantum communication and quantum information processing [1-3], specifically in fibre-based Quantum Key Distribution (QKD) [4-7], which operates mostly at telecom wavelengths; typically, at a wavelength of $1550 \mathrm{~nm}$. The performance of all these applications greatly depends on the parameters of the detector used, such as quantum efficiency,

(c) The Author(s) 2020. This article is licensed under a Creative Commons Attribution 4.0 International License, which permits use, sharing, adaptation, distribution and reproduction in any medium or format, as long as you give appropriate credit to the original author(s) and the source, provide a link to the Creative Commons licence, and indicate if changes were made. The images or other third party material in this article are included in the article's Creative Commons licence, unless indicated otherwise in a credit line to the material. If material is not included in the article's Creative Commons licence and your intended use is not permitted by statutory regulation or exceeds the permitted use, you will need to obtain permission directly from the copyright holder. To view a copy of this licence, visit http://creativecommons.org/licenses/by/4.0/. 
dead time, dark counts, after-pulse probability, gating rate, etc., which need to be metrologically characterized to fully guarantee the reliability of the detection system. Therefore, several national metrological institutes (NMIs), in collaboration with standardisation organisations such as ETSI, are currently putting great efforts into developing novel measurement methods and calibration facilities, enabling the performance of traceable characterization of such detectors by using reference standards [8-11]. From the radiometric point of view, the detection efficiency of such detectors is a key parameter that shall be measured in a traceable manner to the primary standard for optical power (cryogenic radiometer) or by exploiting independent absolute measurement techniques [10, 12-17]. Traceable calibrations of the detection efficiency of free-running Si-SPAD detectors in the visible have been reported by several NMIs claiming relative uncertainties lower than $1.0 \%$ $[8,14,18,19]$. Recently, a comparison on the detection efficiency calibration of a Si-SPAD detector performed between two NMIs using different traceable measurement procedures was presented in [20]. In this paper we report on a preliminary study towards a comparison on the detection efficiency calibration at telecom wavelength of a free-running InGaAs/InP SPAD detector carried out by four European NMIs: CMI, INRIM, NPL and PTB. This study was hosted at INRIM in the autumn of 2017 and CMI, NPL and PTB, brought their equipment there and performed the measurements each over 2 days.

\section{Device under test and measurement principle}

\subsection{Device under test}

In this study, a fibre-coupled indium gallium arsenide (InGaAs/InP) single-photon avalanche diode (SPAD) detector (ID Quantique, ID-220) was used as the device under test (DUT), see Fig. 1. This detector operates in asynchronous detection mode (free-running) within the infrared spectral range from $900 \mathrm{~nm}$ to $1660 \mathrm{~nm}$. Its photon detection probability levels can be adjusted to nominal values of $10 \%, 15 \%$ and $20 \%$, respectively, and its dead time from $1 \mu \mathrm{s}$ to $25 \mu \mathrm{s}$. For this comparison, the detection probability level and the dead time of the detector were configured to be $10 \%$ and $10 \mu \mathrm{s}$, respectively.

To achieve high reproducibility of the measurements, the DUT was operated with a single-mode FC/PC fibre patch cable connected to its input optical port, where the measurement setups of all participating NMIs were connected by means of an FC/PC mating sleeve. Thus, the detection efficiency measured and reported by all participating NMIs

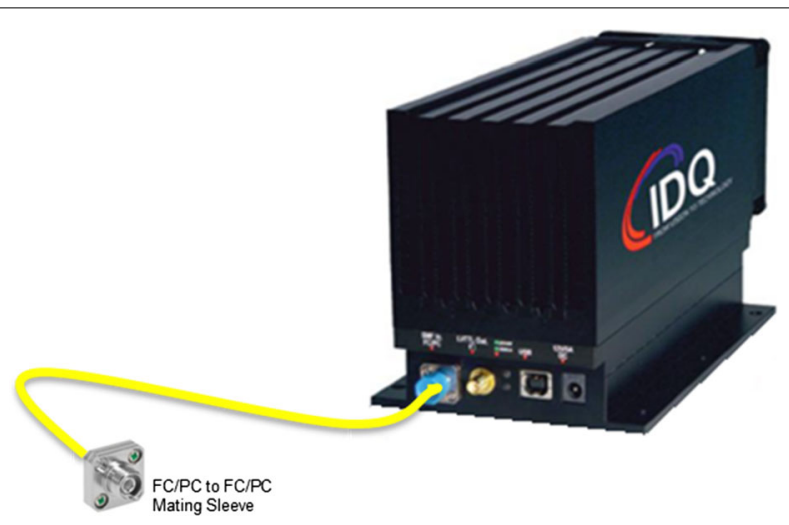

Figure $1 \mathrm{InGaAs/InP}$ free-running single-photon avalanche detector (ID Quantique ID-220 [21]) used as the device under test 
is referenced to this optical connection. The light source used in this study was a sub-ns laser source based on a distributed-feedback laser diode operating at a wavelength of 1550 $\mathrm{nm}$. It was externally triggered to produce sub-nanosecond pulses of approximately $50 \mathrm{ps}$ full-width half maximum (FWHM) at a repetition rate of $110 \mathrm{kHz}$.

\subsection{Measurement principle}

The measurement principle used by all participating NMIs for determining the detection efficiency of the InGaAs/InP SPAD detector was based on the substitution method. This method consists in comparing the optical power, corresponding to the effective number of photons per second, registered by the SPAD detector with the incident mean optical power per laser pulse determined by using a reference analogue detector. A simplified scheme of the setup illustrating this measurement principle is shown in Fig. 2. Typically, a calibrated optical attenuator is used to attenuate the optical power of the laser source down to single-photon levels when irradiating the SPAD detector.

The detection efficiency of the DUT, $\eta_{\text {DUT }}$, is defined as the probability that the DUT detects a photon impinging on it. If one considers an ideal linear detector, $\eta_{\mathrm{DUT}}$ is calculated according to the model:

$$
\eta_{\text {DUT }}=\frac{P_{\mathrm{c}}}{\alpha \cdot P_{0}},
$$

where $P_{0}$ is the optical power with the variable attenuator set to $0 \mathrm{~dB}, \alpha$ is the variable attenuation and $P_{\mathrm{C}}$ is the average optical power corresponding to the effective photon rate measured by the DUT. $P_{C}$ is calculated from the photon rate $\rho$ absorbed by the DUT considering the energy of the photon $h c / \lambda$, that is,

$$
P_{c}=\frac{h \cdot c}{\lambda} \rho
$$

where $h$ is the Planck constant, $c$ is the speed of the light and $\lambda$ is the photon wavelength. Thus, for a pulsed laser source with repetition rate $f_{\text {laser }}$ and mean number of photons $\mu$ per pulse, the value of the photon rate observed by the linear detector is $\rho=f_{\text {laser }} \cdot \mu$. $\eta_{\text {DUT }}$. However, in our specific case the DUT used is not a linear detector, but a threshold (also called "click/noclick") detector, i.e. a detector operated in Geiger mode. Therefore, to retrieve a reliable value of $\eta_{\text {DUT }}$ from the "click" rate, $\rho_{\text {click}}$, counted by our detector, we need to develop a model for its non-linear behavior. Specifically, the probability, $q$,

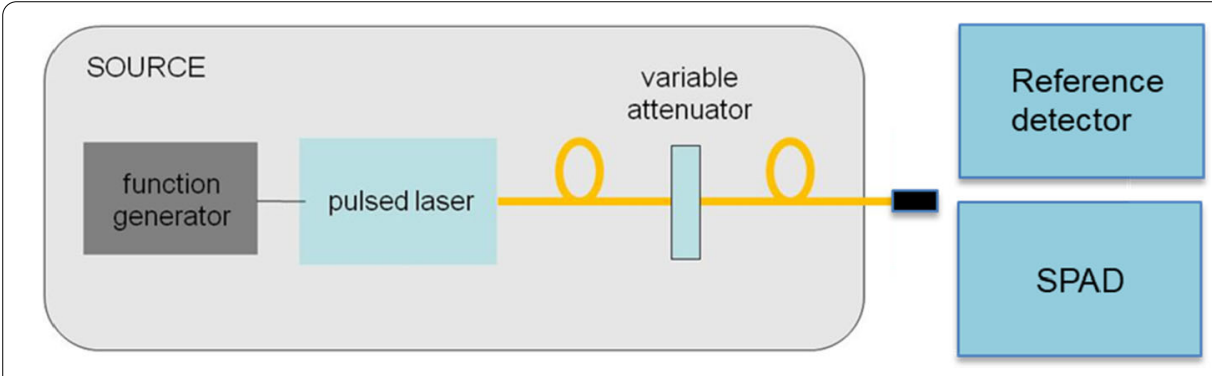

Figure 2 Simplified scheme of the measurement principle (substitution method) used for determining the detection efficiency of a SPAD detector 
of observing a "click" from our detector given an input (Poissonian) laser pulse of mean photon number $\mu$, in the absence of dark counts and dead time is $q=1-e^{-\mu \cdot \eta_{\mathrm{DUT}}}$. Thus the power impinging on the DUT can be expressed in terms of the probability $q$ as

$$
\alpha P_{0}=\frac{h \cdot c}{\lambda} f_{\text {laser }} \mu=-\frac{h \cdot c}{\lambda} f_{\text {laser }} \frac{1}{\eta_{\mathrm{DUT}}} \ln (1-q),
$$

where $q$ can be obtained from the "click" rate $\rho_{\text {click }}=f_{\text {laser }} q$. From Eq. (3) one can easily retrieve $\eta_{\text {DUT }}$. Furthermore, it is interesting to note that Eq. (3) highlights the intrinsic non-linear behavior of this kind of detector.

In more realistic scenarios the probability of having a "click" $q$ must be inferred considering how dark counts $\rho_{\text {dark }}$ and dead time $D$ affect the counting process of the DUT. For this purpose, we developed a proper model of the detection process of the DUT used in this study, which is only valid for the case where $1 / f_{\text {laser }}<D<2 / f_{\text {laser }}$ (for detailed calculation see Appendix, Sect. A.1). The probability $q$ in Eq. (3) is calculated as,

$$
\begin{aligned}
q= & \frac{\rho_{\text {click }}}{f_{\text {laser }}-\rho_{\text {click }}} \\
& +\frac{f_{\text {laser }} \cdot\left(\rho_{\text {click }}-\rho_{\text {click }}^{2} D+f_{\text {laser }} \cdot\left(\rho_{\text {click }} D+\rho_{\text {click }}^{2} \cdot D^{2}-1\right)\right)}{D \cdot\left(f_{\text {laser }}-\rho_{\text {click }}\right)^{2}\left(f_{\text {laser }}-\rho_{\text {click }}+\rho_{\text {click }} \cdot f_{\text {laser }} D\right)} \cdot \rho_{\text {dark }} \cdot D,
\end{aligned}
$$

where $\rho_{\text {click }}$ is the observed "click" rate, $D$ the dead time, $f_{\text {laser }}$ the laser repetition rate and $\rho_{\text {dark }}$ the dark count rate. Note that Eq. (4) is a linearization valid in the limit $\rho_{\text {dark }} \cdot D \ll 1$ (in our experiment $\rho_{\text {dark }} \cdot D \sim 0.01$ ). $\rho_{\text {dark }}$ is estimated from the rate of dark counts $\rho_{\text {click,dark }}^{\prime}$ measured with the pulsed source switched off, and applying the usual dead time correction in the count rate considering a free-running "click/noclick" detector with a fixed deadtime $D$ as $\rho_{\text {dark }}=\frac{\rho_{\text {click,dark }}^{\prime}}{1-\rho_{\text {click,dark }}^{\prime} \cdot D}[22]$.

It should be noted that all parameters contained in Eq. (4) are directly measurable; i.e. no parameter has to be obtained by data fitting, and can be determined with low uncertainty, which makes it a reliable model when assessing the measurement uncertainty of detector detection efficiency.

\section{Measurement method and experimental setup of participating NMIs}

\subsection{CMI}

The experimental setup used by CMI for determining the DUT detection efficiency is shown in Fig. 3. In this setup, the optical power of the short-pulsed laser source is attenuated, down to single-photon levels, by using an optical attenuator Agilent 81571 and a fibre splitter with a nominal ratio of 1:99. The DUT is connected to the branch with $1 \%$ of the optical power, while the branch with $99 \%$ is connected to a low photon flux reference detector for the infrared spectral range ("LOFIR"). The LOFIR consists of a dual-stage thermally cooled Hamamatsu G6805-23 InGaAs photodiode and a highly sensitive lownoise Switched Integrator Amplifier (SIA) using bespoke electronics developed by CMI [23]. Its spectral responsivity was measured using a double monochromator-based facility and a reference detector traceable to the primary standard of the laboratory. In this setup, the splitter ratio must be calibrated, instead of the optical attenuator, to be able to determine the absolute photon flux impinging onto the DUT. Thus, by knowing the splitter ratio and measuring the optical power with the reference detector, the detection 


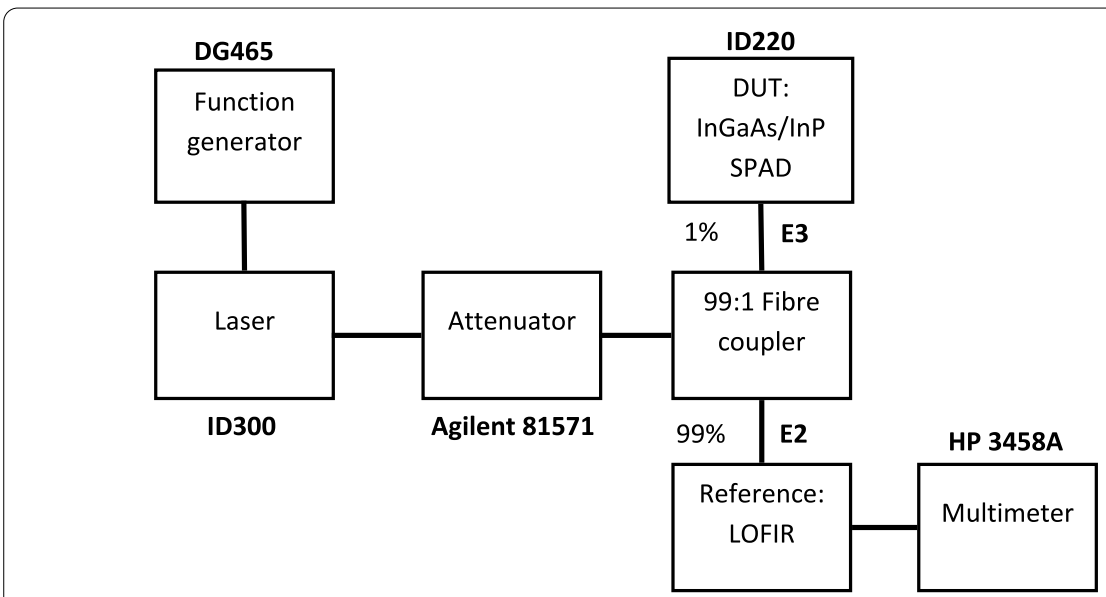

Figure 3 Setup used by CMI for determining the detection efficiency of the InGaAs/InP SPAD detector under test. LOFIR: Low photon flux reference detector for the infrared spectral range

efficiency of the DUT, for each attenuation value of the variable attenuator, is determined by:

$$
\eta_{\text {DUT,CMI }}=-\frac{h \cdot c}{\lambda} \frac{F_{i}}{P_{\text {Lofir } \cdot R_{\text {spl }}}} f_{\text {laser }} \ln (1-q),
$$

where $R_{\text {spl }}$ is the splitting ratio of the splitter, $q$ is calculated using Eq. (4), and $P_{\text {Lofir }}$ is the optical power registered by the LOFIR calculated by,

$$
P_{\text {Lofir }}=\frac{t_{\text {int }} \cdot r_{\text {ref }}}{V_{\text {ref }} \cdot C_{\text {int }}}
$$

where $t_{\text {int }}$ is the LOFIR integration time, $r_{\text {ref }}$ is the responsivity of the LOFIR photodiode, $V_{\text {ref }}$ is the LOFIR output voltage, $C_{\text {int }}$ is the LOFIR integration capacitor. Furthermore, an additional correction factor $F_{i}$ is included in equation (5) to take into account the uncertainty due to the fiber optic connection repeatability. The detailed evaluation model for the estimation of the uncertainty is shown in Appendix A.2.

\subsection{INRIM}

The experimental setup used by INRIM is shown in Fig. 4. In this setup, the optical power of the laser source is attenuated down to single photon levels by using three attenuators: a variable attenuator (Agilent 81571A) and two fixed passive attenuators (FC/APC $10 \mathrm{~dB}$ and 99:1 fibre splitter). The stability of the laser source is monitored by means of a 50:50 beam splitter (point A in Fig. 4) and an optical power meter connected to one of its ports. The attenuated optical beam is coupled to the DUT and the output of the detector is sent to a quad counter (Ortec) connected via GPIB to a PC; counts per second and dark counts per second are acquired by a dedicated acquisition program. The detection efficiency of the DUT is calculated according to the model of Eq. (3):

$$
\eta_{\text {DUT,INRIM }}=-\frac{h \cdot c}{\lambda} \frac{1}{\alpha_{1} \cdot \alpha_{2} \cdot P_{0} \cdot F_{\text {cal }} \cdot F_{i}} f_{\text {laser }} \ln (1-q),
$$




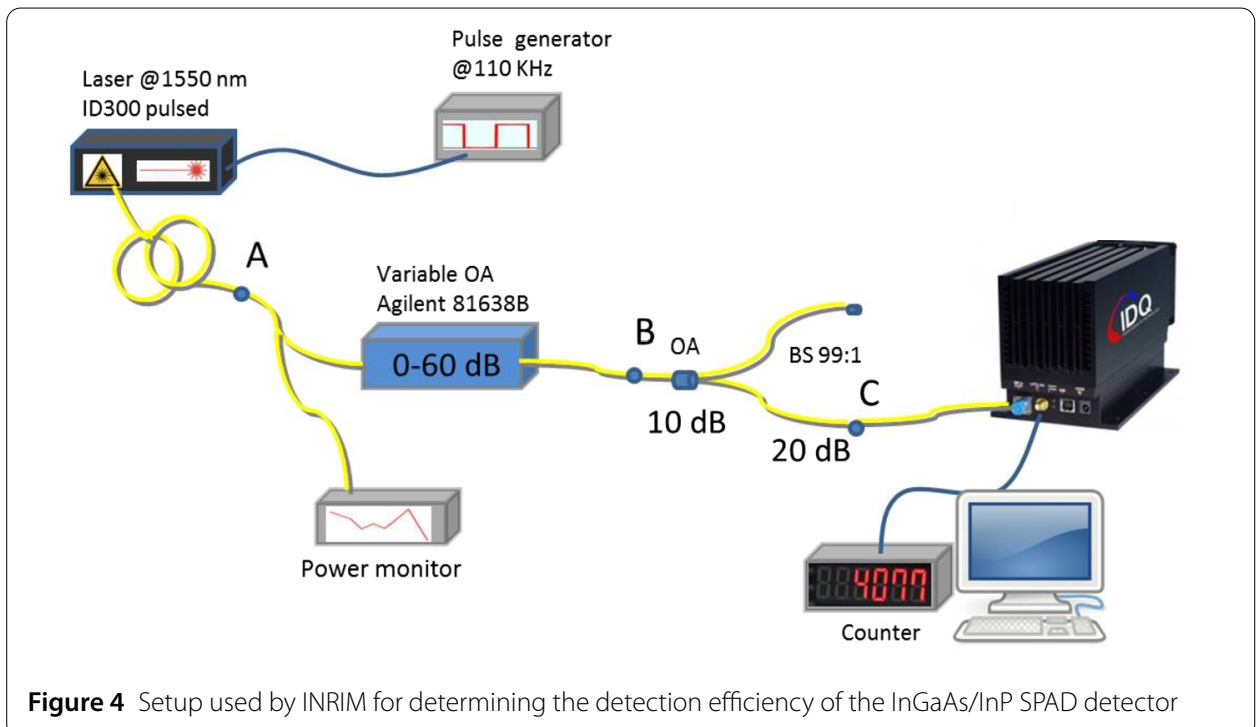

where $P_{0}$ is the power at point B with the variable attenuator set to $0 \mathrm{~dB}, \alpha_{1}$ is the variable attenuation from A to B, $\alpha_{2}$ is the fixed attenuation from B to C (see Fig. 4) and $q$ is calculated according to Eq. (4). $P_{0}, \alpha_{1}$ and $\alpha_{2}$ are evaluated by measuring the average optical power with a power meter (Agilent $81624 \mathrm{~B}$ ) used as reference standard for the optical power measurements and $F_{\text {cal }}$ is the power meter calibration factor. Additional correction factors $F_{i}$ are included for the evaluation of the uncertainty such as nonlinearity of the power meter and fibre connector repeatability (see Appendix A.2)

\subsection{NPL}

The experimental setup used by NPL is shown in Fig. 5. In this setup, the short-pulsed laser source is attenuated to the single-photon level by means of two attenuators: a manually adjustable Thorlabs VOA50-FC, and a calibrated electronically-controlled attenuator HP 8158B. The optical power of the source is monitored via a 99:1 fibre splitter and a monitor detector. The reference standard used is an HP 8153A optical power meter calibrated against an integrating sphere transfer standard, which is traceable to the primary standard for optical power (cryogenic radiometer) of the laboratory. Its linearity is measured using an optical fibre implementation of the double aperture technique, down to 1 $\mathrm{pW}$. The power meter is also used to calibrate the attenuator.

In this setup, the DUT counts per laser pulse were recorded in a synchronous manner, that is, the function generator outputs an identical clock pulse on two outputs (A and B) which are used to drive the laser and timer/counter respectively. The timer/counter acts as a stopwatch receiving a synchronous 'start' signal from the function generator and a 'stop' signal from the DUT. The time interval between these two events was recorded into $100 \mathrm{ps}$ wide time-bins. The counter was operated in multi-stop mode and set to record stops occurring in a window of $16 \mu$ s subsequent to a 'start'. This process was repeated for 10 million 'start' signals to accumulate time-stamped data. The data was histogrammed as a function of time-delay with respect to the 'start' pulse, and the effective count rate per second was calculated by summing all histogram counts within a laser period to facilitate comparison with the other participants. 


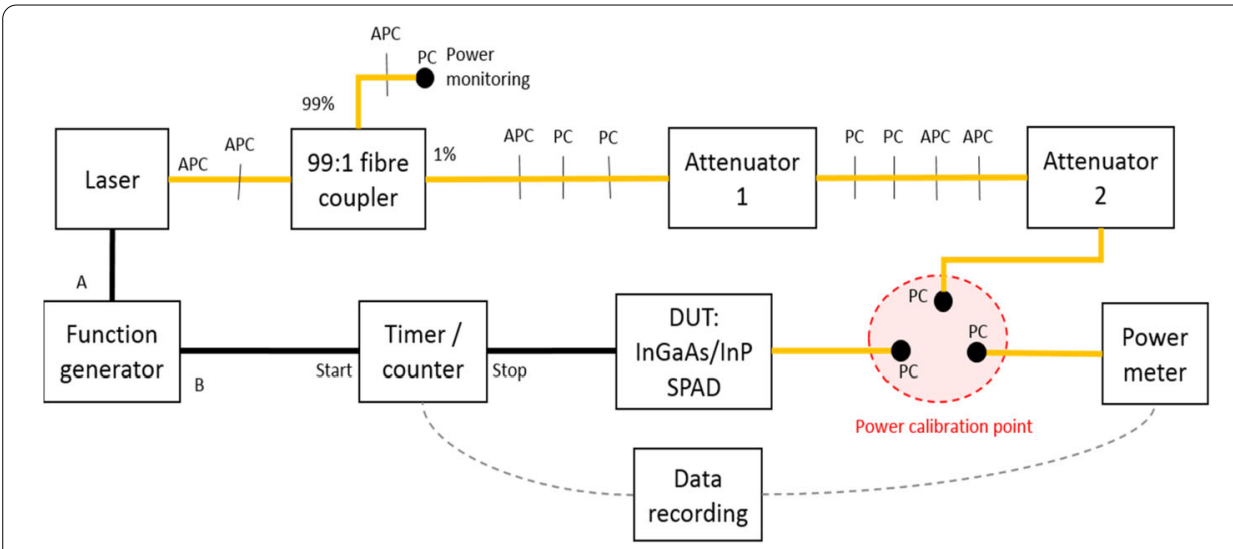

Figure 5 Setup used by NPL for measuring the detection efficiency of the InGaAs/InP SPAD detector. All fibre-to-fibre connections used in the experiment are shown. The red circled area denotes fibre connections that are swapped between successive power calibration measurements and detection efficiency measurements

The detection efficiency of the DUT is determined by,

$$
\eta_{\mathrm{DUT}, \mathrm{NPL}}=-\frac{h \cdot c}{\lambda} \frac{1}{P_{\mathrm{cal}} \cdot \alpha \cdot F_{\mathrm{cal}} \cdot S \cdot F_{i}} f_{\text {laser }} \ln (1-q),
$$

where $P_{\text {cal }}$ is the optical power measured by the reference power meter at the power calibration point with Attenuator 2 set to its minimum value $\left(\alpha_{\text {ref }}=1\right)$ and high laser repetition rate $\left(f_{\text {laser }}=2 \mathrm{MHz}\right), S$ is a scaling factor which takes into account the change of the laser pulse shape that occurs at different laser repetition rates, $F_{\text {cal }}$ is the scaling (calibration) factor to be applied to power meter reading. Moreover, additional correction factors $F_{i}$ such as nonlinearity of the optical power measurements, stability of the power meter spectral responsivity and fibre connector repeatability are included for the evaluation of the uncertainty. The detailed evaluation model is shown in Appendix A.2.

\subsection{PTB}

Figure 6 shows the experimental setup used by PTB. Here, the optical power of the shortpulsed laser source is strongly attenuated by means of two attenuators (Agilent 81571A), which are previously calibrated at higher optical power levels using the double attenuator technique [8]; i.e. their attenuation factors, $\alpha_{1}$ and $\alpha_{2}$, are separately determined with a low noise analogue InGaAs/InP photodiode (Hamamatsu G8605-23), cooled at $-20^{\circ} \mathrm{C}$, in a sequential way for different attenuation levels. This InGaAs/InP photodiode is also used as reference detector for the optical power measurement required to determine the mean photon number per laser pulse during the calibration process. Its absolute responsivity was determined via a calibration against a thermopile traceable to PTB's primary standard for optical power (cryogenic radiometer) at a wavelength of $1550 \mathrm{~nm}$. Moreover, the linearity of this photodiode in combination with the Femto/Picoammeter (Keysight B2981A), used for the measurement of the photocurrent, was characterized for the optical power range from $100 \mu \mathrm{W}$ to $1 \mathrm{pW}$. Thus, from the measurement of the effective count rate of the DUT and the determined average optical power impinging onto it, the detection efficiency of the DUT is determined by,

$$
\eta_{\mathrm{DUT}, \mathrm{PTB}}=-\frac{h \cdot c}{\lambda} \frac{F_{i}}{P_{0, \mathrm{InGaAs}} \cdot\left(1+F_{\mathrm{Lin}}\right) \cdot \alpha_{1} \cdot \alpha_{2}} f_{\text {laser }} \ln (1-q) .
$$




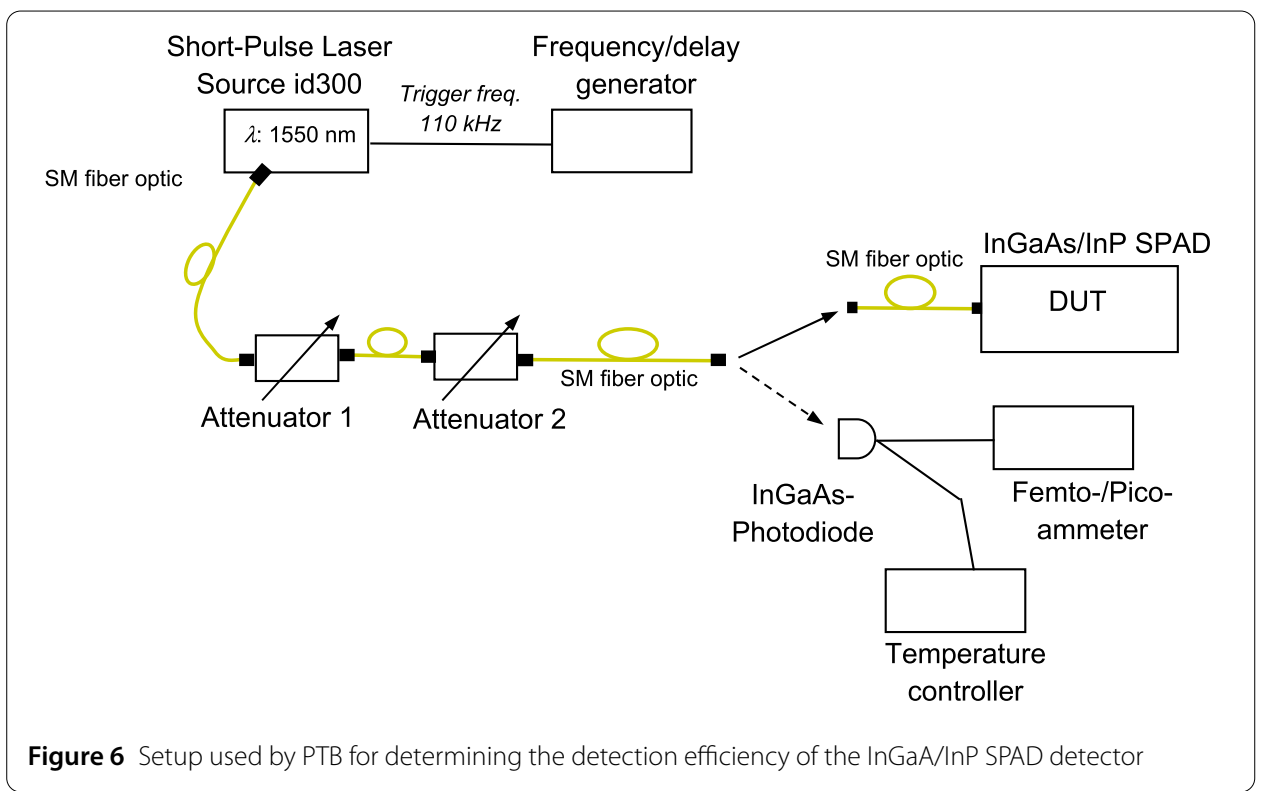

$P_{0, \text { InGaAs }}$ is the optical power measured with the InGaAs reference detector with the variable attenuators (Attenuator 1 and 2 ) set to $0 \mathrm{~dB}, F_{\mathrm{Lin}}$ is the linearity correction factor of the InGaAs reference detector and $F_{i}$ is the correction factor corresponding to the fibre connector repeatability. The detailed model used for the evaluation of the uncertainty is shown in Appendix A.2.

\section{Results and analysis}

Figure 7 shows the detection efficiency of the DUT determined by all NMIs participants for a mean photon number per pulse $\mu$ between 0.01 and 2.4, which corresponds to a photon rate of approximately 1100 photon/s and 193,000 photon/s, respectively. The measurement values shown in green correspond to the detection efficiency obtained by correcting the SPAD dead time, while in red without correction. The uncertainty associated to each measurement value, shown with error bars, was calculated following the guidelines described in [24]. As can be observed, the detection efficiency without detector dead time correction (considering an indicative mean value of $\eta_{\text {DUT }}=10.419 \pm 0.001$ for $\mu<0.15$ ) decreases for mean photon numbers higher than $\sim 0.1$. In general, it is observed that the dead time model of the photon-counting process given in equation (4) corrects well the non-linear detection response of the SPAD detector for mean photon numbers up to $\sim 2.4$. Moreover, the measurement uncertainty increases at low mean photon number; see for example the uncertainty of the detection efficiency measured by NPL at $\mu \sim 0.01$. This is because of the high contribution of the Poissonian noise of the SPAD dark counts ( $\sim 900$ count/s) to the counted photons at this photon rate levels. The detailed uncertainty budget reported by each participant is shown in Appendix A.2. It should be noted that the corrected detection efficiency values in Fig. 7 show a significant correction $(\sim 2 \%)$ already for small mean photon numbers. This is caused by the high contribution of dark counts and their associated dead time effect on the detection process for this kind of detector, when operated with a pulsed laser source with a period $\tau<D<2 \tau$.

A slightly noticeable under-correction of the efficiency starts to be evident at higher mean photon numbers $\mu$ (typically, i.e. when the click rate starts rising because of the non- 


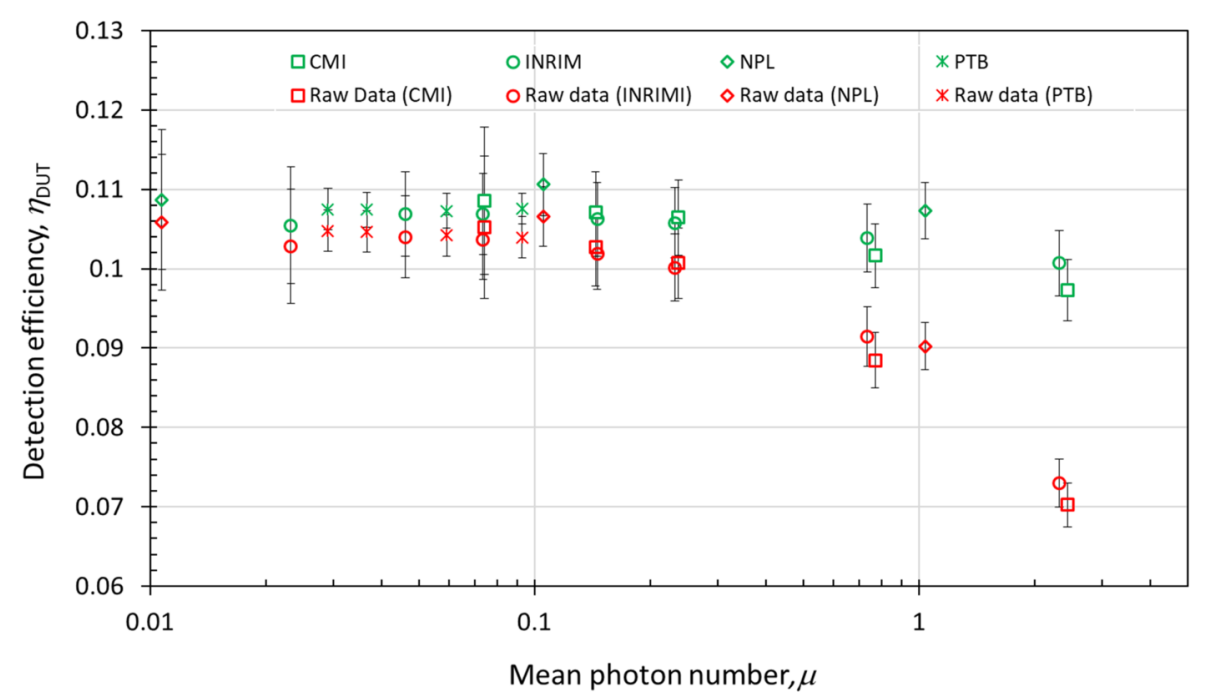

Figure 7 Detection efficiency of the InGaAs/InP SPAD detector measured by all participants between the mean photon number per pulse from 0.01 and 2.4, which correspond to a photon rate of approximately 1100 photon/s and 193,000 photon/s for a wavelength of $1550 \mathrm{~nm}$, respectively. The data in red are referred to raw data; i.e. without correcting the SPAD dead time, and the green are the corrected data according to equation (4). Error bars: expanded uncertainty $(k=2)$

negligible multi-photon component). This can be explained by the presence of afterpulses during the SPAD detection resulting in more than one signal per incident pulse. It has been observed that in InGaAs detectors afterpulses increases with the number of counts and may last for several $\mu \mathrm{s}$ [25]; their presence can result in a reduction of the apparent detection efficiency due to the increase of the overall dead time. Nevertheless, a proper characterization and model of afterpulses is beyond the scope of the study, also because for the DUT the model for the estimation of detection efficiency is rather robust in the whole interval of the pulse energy explored, that, was as wide as more than two orders of magnitude. Extending measurements to such a wider energy interval appears not to be necessary in view of a comparison, but we prefer to also test the validity and limitations of our measurement model outside the study mean photon number range.

In this study, the reproducibility and the insertion losses of the fibre-to-fibre coupling through the FC/PC mating sleeve on the SPAD detection efficiency was not extensively investigated by all participants during the comparison, as e.g. in $[19,26]$. Instead, a value of $0.5 \%$ for the uncertainty associated with the reproducibility of the FC/PC fibre connexion, determined by only one participant in a separate setup, was used by all participants in this study. As shown in Fig. 9, with this value acceptable consistency between the measurement results is obtained.

To quantify the mean between the detection efficiency measurements reported by all participants, shown in green in Fig. 7, their measurement values were first averaged for each participant; i.e. a "participant" detection efficiency $\eta_{p}$ and its uncertainty for each participant was first calculated. The obtained averaged values and their associated uncertainty are shown in Table 1 . Then, using these averaged values, the weighted mean value $\bar{\eta}_{p, w}$ and its associated uncertainty $U\left(\bar{\eta}_{p, w}\right)$ were calculated according to [27], that is,

$$
\bar{\eta}_{p, w}=\left(\sum_{i=1}^{n} \eta_{p, i} \cdot w_{i}\right) / \sum_{i}^{n} w_{i}
$$


Table 1 Average and expanded uncertainty of the detection efficiency reported by each participant laboratory for the mean photon per pulse between 0.01 and 2.4 ( 1100 photon/s and 193,000 photon/s)

\begin{tabular}{lll}
\hline Laboratory & Participant detection efficiency, $\eta_{p}$ & Expanded Uncertainty, (rel.) \\
\hline CMI & 0.1043 & $0.0056(5.3 \%)$ \\
INRIM & 0.1052 & $0.0050(4.8 \%)$ \\
NPL & 0.1090 & $0.0054(5.0 \%)$ \\
PTB & 0.1075 & $0.0024(2.7 \%)$ \\
\hline
\end{tabular}

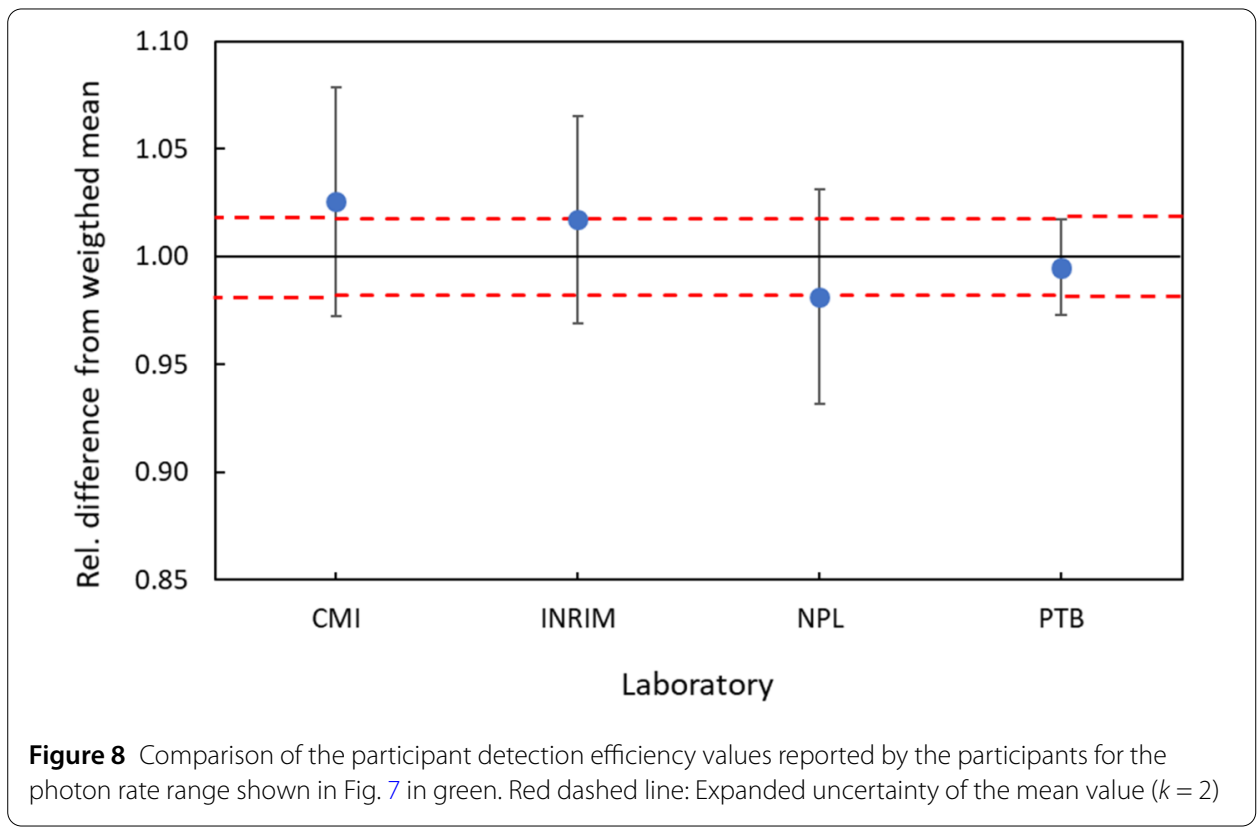

with $w_{i}=U\left(\eta_{p, i}\right)^{-2}$

$$
U\left(\bar{\eta}_{p, w}\right)=\frac{1}{\sqrt{\sum_{i=1}^{n} \frac{1}{U\left(\eta_{p, i}\right)^{2}}}},
$$

where $n$ is the number of participating laboratories.

The mean weighted value $\bar{\eta}_{p, w}$ and its corresponding expanded uncertainty obtained are:

$$
\begin{aligned}
\bar{\eta}_{p, w} & =0.1070 \pm 0.0019 \\
& =0.1070 \pm 1.8 \% .
\end{aligned}
$$

The relative difference of the participant detection efficiency values from the weighted mean is shown in Fig. 8. The dashed lines denote the relative expanded uncertainty of the weighted mean. Here it can be observed that the maximum relative difference between the values of the participant's detection efficiency and the weighted mean is less than $2.6 \%$. In addition, to determine the consistency of the measurements, the $E_{n}$ values were calculated 


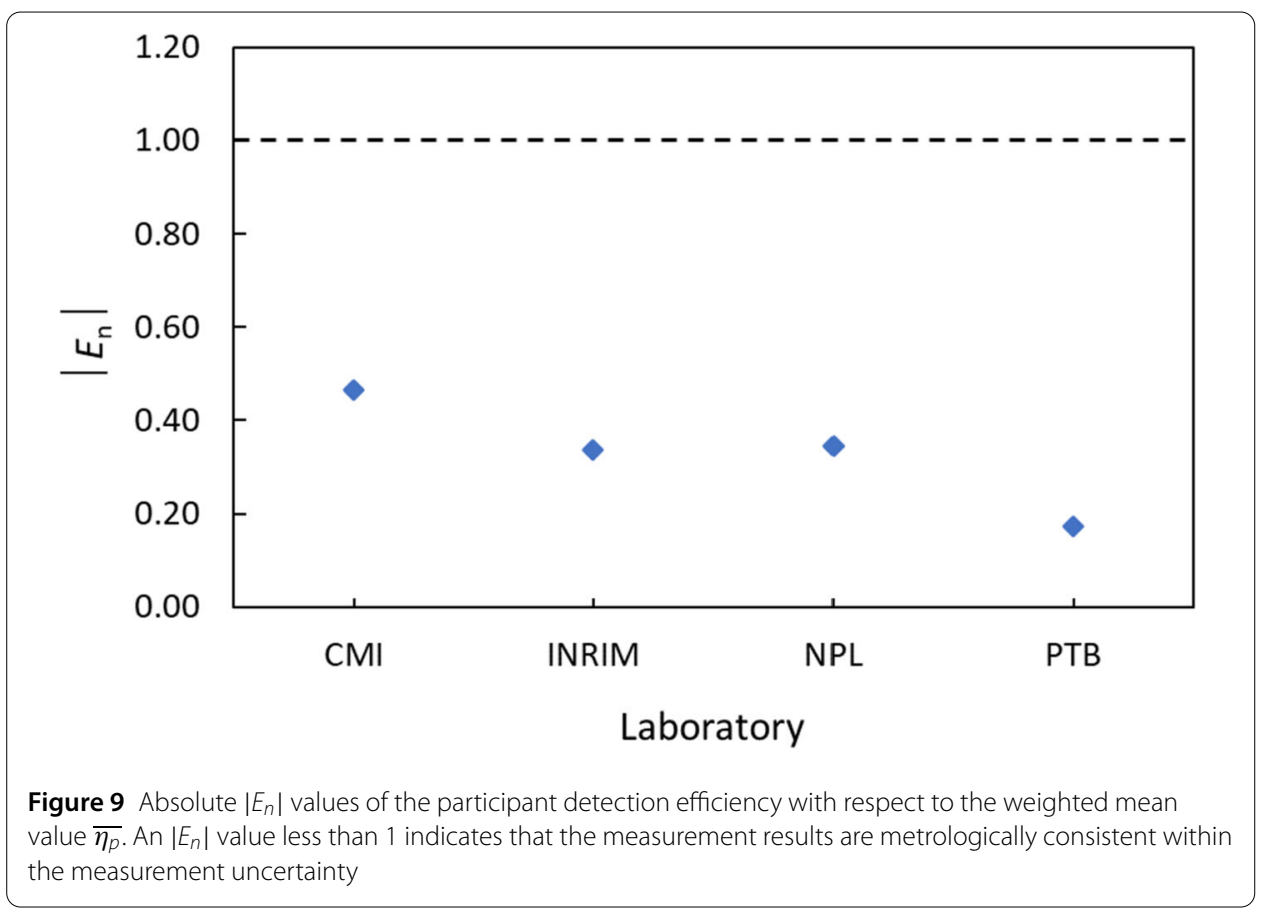

according to [28],

$$
E_{n, i}=\frac{\eta_{p, i}-\bar{\eta}_{p, w}}{\sqrt{U\left(\eta_{p, i}\right)^{2}+U\left(\bar{\eta}_{p, w}\right)^{2}}} .
$$

An $\left|E_{n}\right|$ value $<1$ means that the measurement results are metrologically consistent within the measurement uncertainty. In this study, the obtained $\left|E_{n}\right|$ values are smaller than 0.6 for all participating laboratories, see Fig. 9 , indicating the consistency of the measurements.

\section{Conclusions}

A detailed study to develop a realistic measurement model for the detection efficiency of a fibre-coupled free-running InGaAs/InP single photon detector measured with the substitution method against a pulsed laser was carried out by four national metrology institutes (CMI, INRIM, NPL and PTB) using different experimental setups and reference standards. Specifically, the evaluation of the detection efficiency included a correction for the dead time of the device under test allowing a faithful extension of the measurement range over three orders of magnitude (in terms of mean photon number), demonstrating the consistency of the measurement model developed. The $E_{n}$ values derived for each participant are less than 0.5 , which means that the measurement results are consistent within the stated measurement uncertainty. This study opens a path towards future international comparison of the detection efficiency of single-photon detectors at telecom wavelength. This is of utmost importance for quantum photonics technology applications in general, and for quantum communications in particular, since these detectors are at the core technology of quantum key distribution systems. 


\section{Appendix}

\section{A.1 Dead time correction model}

To infer the value of the corrected photon rate $\lambda_{\text {corr }}$ from the counted photons, we need to apply appropriate corrections that consider how dark counts and dead time $D$ affect the counting process considering that we employed a free running single-photon detector (Geiger-mode operation) and a pulsed laser source with repetition rate $f_{\text {laser }} . \mu$ is the mean number of photons per pulse impinging on the detector and, in the absence of dark counts and dead times, the probability of having a "click" per laser pulse is $q=1-e^{-\mu \eta}$.

Let us first consider the dead time, still in the case of the absence of dark counts, in a free running detector that measures a pulsed laser operating with a period $\tau<D<2 \tau$, ${ }^{\text {a }}$ with $\tau=1 / f_{\text {laser }}$ (see Fig. A1). The true probability of having a "click" is [29]:

$$
p_{\text {click }}=q(1-q(1-q(1-q \cdots)))=\frac{q}{q+1} \text {. }
$$

This expression accounts for the fact that the probability of observing a "click" from a pulse requires that the previous pulse should not have produced a "click". Next, we have to consider the contribution of dark counts. Since the detector operates in free running mode, spurious counts are present at every time during and in absence of the laser pulse (see Fig. A1). To account for this, we include in the model the probability $p_{\text {dark }}(0)$ of having no dark counts in the dead time period $D$. If the dark count rate, in absence of dead time, is $\rho_{\text {dark }}$, the probability of having a "click" in the laser pulse duration is therefore:

$$
p_{\text {click,true }}=p_{\text {dark }}(0) \frac{q}{q+1}
$$

with $p_{\text {dark }}(0)=e^{-\rho_{\text {dark }} D}$, which assumes that it behaves according to a Poissonian process. This condition is a simplification and holds only if the probability of having a dark count in the dead time period is low; i.e. if $1-p_{\text {dark }}(0) \ll 0.1$. In our experiment it was typically less than 0.01 .

The effective "click" rate of the detection is the sum of the "click" rate due to photons present in each pulse and the photons of dark counts $\rho_{\text {click,dark: }}$ :

$$
\rho_{\text {click }}=\rho_{\text {click,true }}+\rho_{\text {click,dark }},
$$

where $\rho_{\text {click,true }}=f_{\text {laser }} p_{\text {click,true }}$.

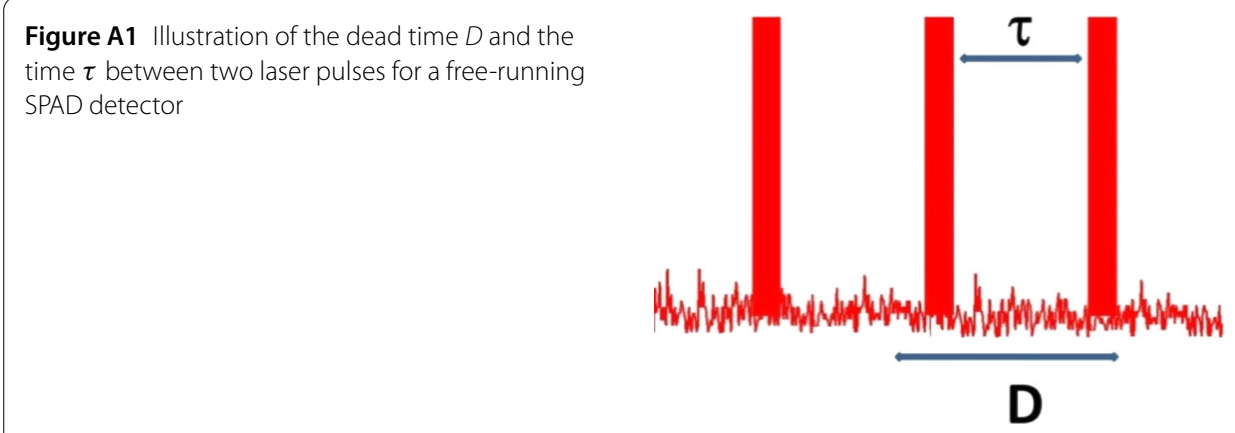


The rate of dark counts $\rho_{\text {click,dark }}$ should account for the presence of the dead time. The correction is the "usual-one" when a Poissonian process is considered in the presence of the detector dead time: ${ }^{\mathrm{b}}$

$$
\rho_{\text {click }, \text { dark }}=\frac{\rho_{\text {dark }}}{1+\left(f_{\text {laser }} q+\rho_{\text {dark }}\right) D} .
$$

Note that the additional term $f_{\text {laser }} q$ accounts for the fact that also "true" counts increase the off-time of the detectors; i.e. they arrive periodically in time, but the time randomness of dark counts guarantees that their effect is indistinguishable from the ones of the dark counts themselves.

By substituting the dead time corrected counts and dark counts rate in the expression of the effective "click" rate, we have:

$$
\rho_{\text {click }}=f_{\text {laser }} e^{-\rho_{\text {dark }} D} \frac{q}{q+1}+\frac{\rho_{\text {dark }}}{1+\left(f_{\text {laser }} q+\rho_{\text {dark }}\right) D} .
$$

By rearranging this formula, we can infer the value of $q$. In the limit $\rho_{\text {dark }} D \ll 1$ and at the first order of approximation we obtain:

$$
q=\frac{\rho_{\text {click }}}{f_{\text {laser }}-\rho_{\text {click }}}+\frac{f_{\text {laser }}\left(\rho_{\text {click }}-\rho_{\text {click }}^{2} D+f_{\text {laser }}\left(\rho_{\text {click }} D+\rho_{\text {click }}^{2} D^{2}-1\right)\right)}{D\left(f_{\text {laser }}-\rho_{\text {click }}\right)^{2}\left(f_{\text {laser }}-\rho_{\text {click }}+\rho_{\text {click }} f_{\text {laser }} D\right)} \rho_{\text {dark }} D .
$$

All the quantities in Eq. (A.6), $\rho_{\text {click }}, D$ and $f_{\text {laser }}$, are measured except $\rho_{\text {dark }}$. To estimate $\rho_{\text {dark }}$, we should switch-off the pulsed laser source and measure the rate of dark counts $\rho_{\text {dark }, D}$. According to Eq. (A.4), the rate of counted dark counts will be

$$
\rho_{\text {dark }, D}=\frac{\rho_{\text {dark }}}{1+\rho_{\text {dark }} D}
$$

Thus, inverting Equation (A.7), $\rho_{\text {dark }}$ will be estimated as

$$
\rho_{\text {dark }}=\frac{\rho_{\text {dark }, D}}{1-\rho_{\text {dark }, D} D}
$$

The number of the corrected count rate, used by all participant in this comparison to estimate the efficiency of the detector, is therefore:

$$
\rho_{\text {corr }}=f_{\text {laser }} \mu \eta=-f_{\text {laser }} \ln (1-q) .
$$

Figure A2 shows the effect of this correction on the detection efficiency of the DUT determined by all participants. The red markers represent the calculated detection efficiency using data only corrected by background photons while the green markers are the values of the efficiency after the dead time correction. The model (A.9) corrects the effect of dead time over three order of magnitude of mean photon number $\mu$ and can be applied to extend the comparison of the detection efficiency measurement to a wide range of photon counts.

A slightly noticeable under-correction of the efficiency starts to be evident at higher mean photon numbers $\mu$ (typically, i.e. when the click rate starts rising because of the nonnegligible multi-photon component). This can be explained by the presence of afterpulses 


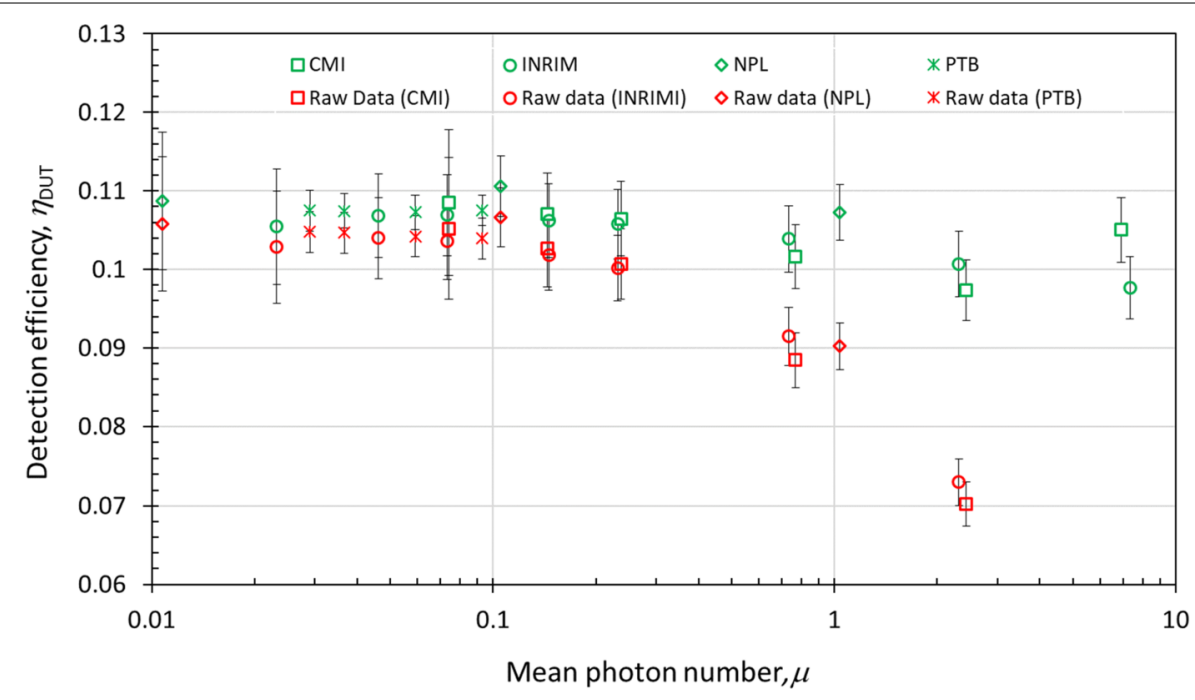

Figure A2 Detection efficiency $\eta_{\text {DUT }}$ of the DUT as function of the mean photon number per pulse. Red data are referred to raw data; i.e. without correcting the SPAD dead time, and green are corrected data according to Eq. (A.9). Error bars: expanded uncertainty of the measurement

during the SPAD detection resulting in more than one signal per incident pulse. It has been observed that in InGaAs detectors afterpulses increases with the number of counts and may last for several $\mu \mathrm{s}$ [25]; their presence can result in a reduction of the apparent detection efficiency due to the increase of the overall dead time. Nevertheless, a proper characterization and model of afterpulses is beyond the scope of the study, also because for the DUT the model for the estimation of detection efficiency is rather robust in the whole interval of the pulse energy explored, that, was as wide as more than two orders of magnitude. Extending measurements to such a wider energy interval appears not to be necessary in view of a comparison, but we prefer to also test the validity and limitations of our measurement model outside the study mean photon number range.

\section{A.2 Evaluation model and uncertainty budget}

\section{A.2.1 CMI}

The model used by CMI for the evaluation of the standard uncertainty of the detection efficiency measurement is given by,

$$
\eta_{\mathrm{DUT}, \mathrm{CMI}}=\frac{h \cdot c}{\lambda} \cdot \frac{\rho_{\text {corr }}}{R_{\mathrm{spl}} \cdot P_{0, \mathrm{LOFR}}} \cdot F_{\mathrm{fconn}}
$$

where $h \cdot c / \lambda$ is the photon energy, $\rho_{\text {corr }}$ are the corrected counts ("clicks") obtained by using the correction model derived in Sect. A.1, which includes the measured count rate $\rho_{\text {click }}$, the dark counts $\rho_{\text {Dark }, \text { click }}$ and dead time $D$ of the SPAD detector as well as the laser frequency $f_{\text {laser }} . P_{0, \mathrm{LOFR}}$ is the optical power measured by the LOFIR, $R_{\mathrm{spl}}$ is the splitting ratio of the splitter and $F_{\text {fconn }}$ is the correction factor that takes into account the uncertainty associated to the fibre optic connection repeatability; connecting to and disconnecting from the SPAD and power meter.

The uncertainty sources related to the evaluation model (A.10) are shown in Table A1. 


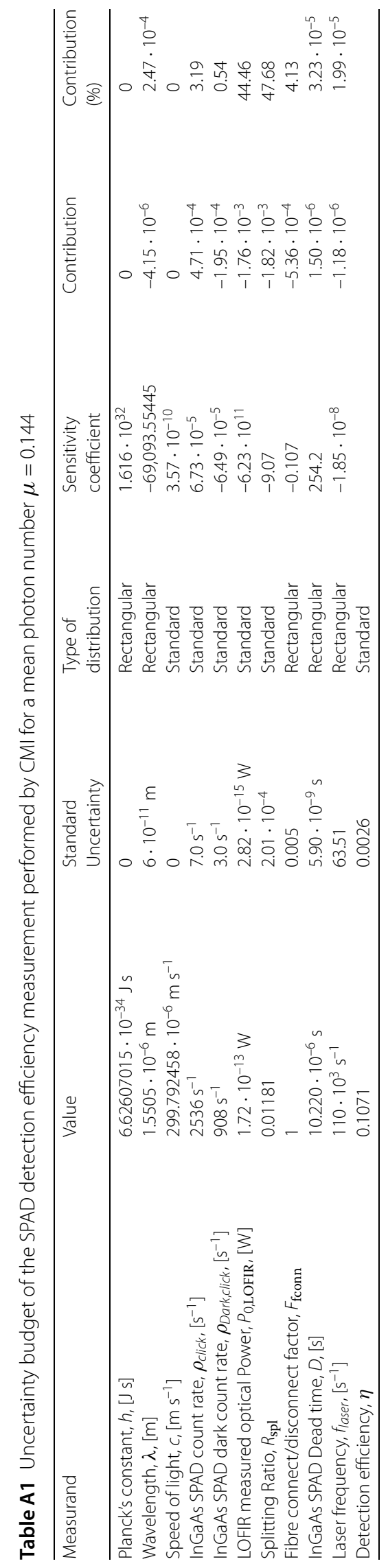




\section{A.2.2 INRIM}

The model used by INRIM for the evaluation of the standard uncertainty of the detection efficiency measurement is given by,

$$
\eta_{\text {DUT,INRIM }}=\frac{h \cdot c}{\lambda} \cdot \frac{\rho_{\text {corr }}}{\alpha \cdot P_{0} \cdot F_{\text {cal }}} \cdot F_{\text {fconn }},
$$

where $h \cdot c / \lambda$ is the photon energy, $\rho_{\text {corr }}$ are the corrected counts ("clicks") obtained by using the correction model derived in Sect. A.1, which includes the measured count rate $\rho_{\text {click }}$, the dark counts $\rho_{\text {Dark click }}$ and dead time $D$ of the SPAD detector as well as the laser frequency $f_{\text {laser }} . P_{0}$ is the power measured at the point $B$ with the variable attenuator set to 0 $\mathrm{dB}, \alpha$ is the total attenuation factor, $F_{\text {cal }}$ is the calibration factor of the optical power meter (Agilent $81624 \mathrm{~B}$ ). $F_{\text {fconn }}$ is the correction factor that takes into account the uncertainty associated to the fibre optic connection repeatability.

The uncertainty sources related to the evaluation model (A.11) are shown in Table A2.

\section{A.2.3 NPL}

The model used by NPL for evaluating the standard uncertainty of the detection efficiency measurement is given by,

$$
\begin{aligned}
\eta_{\mathrm{DUT}, \mathrm{NPL}}= & \frac{h \cdot c}{\lambda} \cdot \frac{\rho_{\text {corr }}}{\left(P_{\mathrm{cal}}-P_{\mathrm{Dark}}\right) \cdot \alpha \cdot F_{\mathrm{cal}} \cdot S \cdot\left(1+F_{\text {lin }}\right) \cdot\left(1+F_{\mathrm{stab}}\right) \cdot\left(1+F_{\lambda}\right)} \\
& \cdot F_{\text {fconn }},
\end{aligned}
$$

where $h \cdot c / \lambda$ is the photon energy, $\rho_{\text {corr }}$ are the corrected counts ("clicks") obtained by using the correction model derived in Sect. A.1, which includes the measured count rate $\rho_{\text {click }}$, the dark counts $\rho_{\text {Dark,click }}$ and dead time $D$ of the SPAD detector as well as the laser frequency $f_{\text {laser }} . P_{\text {cal }}$ is the optical power measured by the reference power meter, $P_{\text {Dark }}$ is the power meter dark offset, $S$ is a scaling factor which takes into account the change of the laser pulse shape that occurs at different laser repetition rates, $F_{\text {cal }}$ is the scaling (calibration) factor to be applied to power meter readings, $F_{\text {lin }}$ is the nonlinearity correction factor of the optical power measurement, $F_{\text {stab }}$ is the fractional change in $F_{\text {cal }}$ between calibration and comparison measurements (power meter instability), $F_{\lambda}$ is the fractional change in (responsivity) scaling factor due to error in wavelength and $F_{\text {fconn }}$ is the correction factor which takes into account the uncertainty associated to the fibre optic connection repeatability.

The uncertainty sources related to the evaluation model (A.12) are shown in Table A3. The power meter readings were performed at a laser repetition rate of $2 \mathrm{MHz}$.

\section{A.2.4 PTB}

The model used by PTB for evaluating the standard uncertainty of the detection efficiency measurement is given by,

$$
\eta_{\mathrm{DUT}, \mathrm{PTB}}=\frac{h \cdot c}{\lambda} \cdot \frac{s_{\mathrm{InGaAs}} \cdot \rho_{\text {corr }}}{I_{\mathrm{ph}} \cdot\left(1+F_{\mathrm{Lin}}\right) \cdot \alpha} \cdot F_{\mathrm{fconn}},
$$

where $h \cdot c / \lambda$ is the photon energy, $\rho_{\text {corr }}$ are the corrected counts ("clicks") obtained by using the correction model derived in Sect. A.1, which includes the measured count rate 


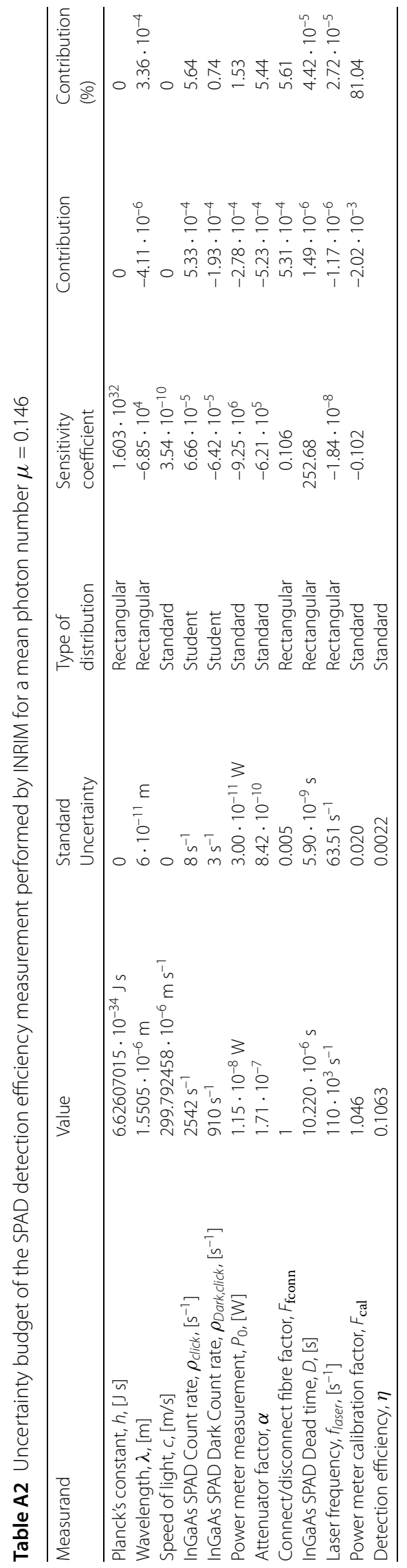




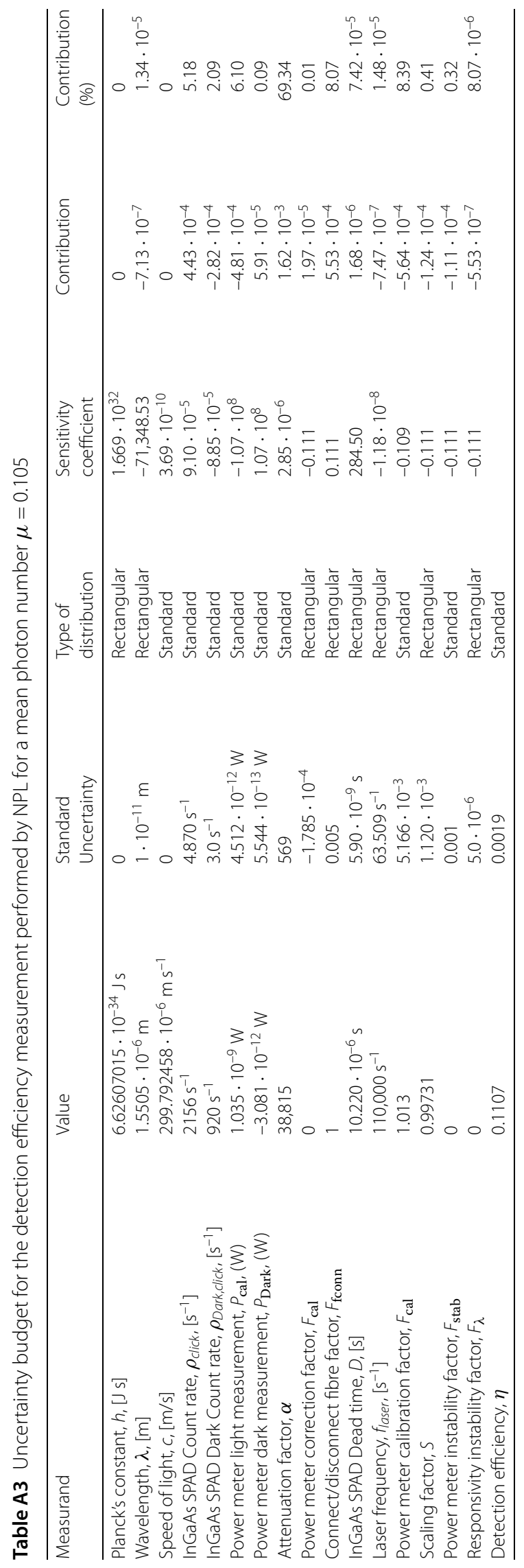




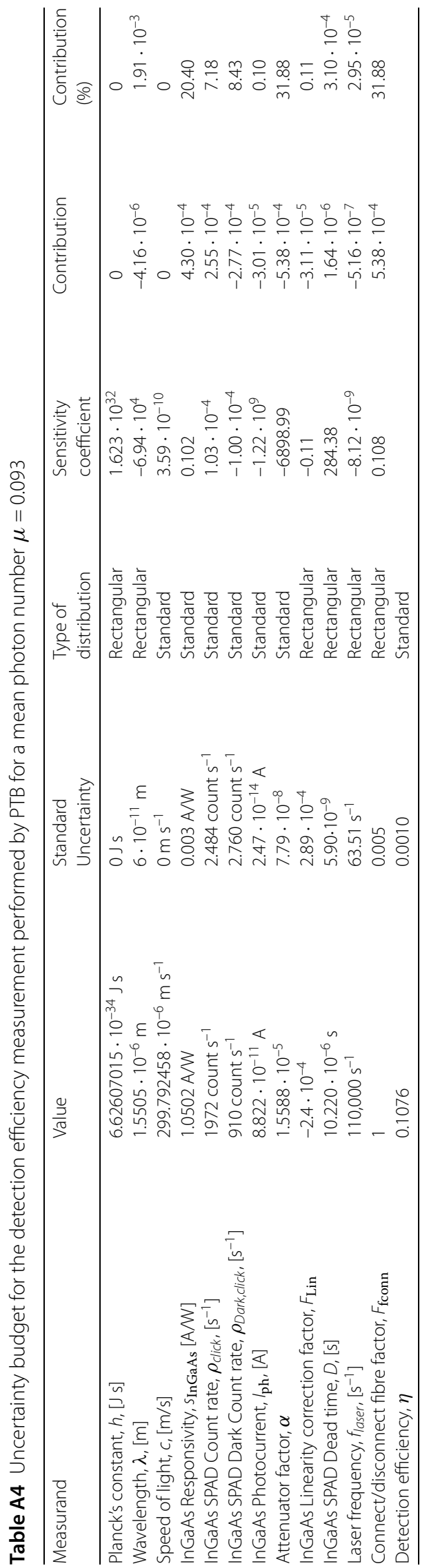


$\rho_{\text {click }}$, the dark counts $\rho_{\text {Dark,click }}$ and dead time $D$ of the SPAD detector as well as the laser frequency $f_{\text {laser }}$. $S_{\text {InGaAs }}$ is the responsivity of the InGaAs analogue reference detector, $I_{\mathrm{ph}}$ is the measured photocurrent generated by the InGaAs reference detector, $F_{\text {Lin }}$ is the linearity correction factor of the InGaAs reference detector, $\alpha$ is the total attenuation factor and $F_{\text {fconn }}$ is the correction factor that takes into account the uncertainty associated to the fibre optic connection repeatability.

The uncertainty sources related to the evaluation model (A.13) are shown in Table A4.

\section{Acknowledgements}

The work reported on this paper was funded by project EMPIR-14IND05 "MIQC2" and EMPIR-17FUN06 "SIQUST". These projects received funding from the EMPIR program co-financed by the Participating States and from the European Union Horizon 2020 research and innovation program.

\section{Funding}

This work was funding by the European Metrology Programme for Innovation and Research (EMPIR). Projects: EMPIR-14IND05 "MIQC2" and EMPIR-17FUN06 "SIQUST". Open Access funding enabled and organized by Projekt DEAL.

\section{Abbreviations}

CMI, Cesky Metrologicky Institut; DUT, Device under test; FWHM, full-width half maximum; INRIM, Istituto Nazionale di Ricerca Metrologica; LOFIR, Low photon flux reference detector for the infrared spectral range; NMI, national metrology institute; NPL, National Physical Laboratory; PTB, Physikalisch-Technische Bundesanstalt; QKD, Quantum Key Distribution; SPAD, single-photon avalanche diode.

\section{Availability of data and materials}

Data are available under request.

\section{Competing interests}

Authors declare non-financial competing interests.

\section{Authors' contributions}

The main idea of this paper was proposed by ML, AM and IPD. ML prepared the manuscript initially and performed all the steps of the proofs in this research. All authors read and approved the final manuscript.

\section{Author details}

${ }^{1}$ Physikalisch-Technische Bundesanstalt (PTB), Bundesallee 100, 38116 Braunschweig, Germany. ${ }^{2}$ Istituto Nazionale di Ricerca Metrologica (INRIM), Strada delle Cacce 91, I-10135 Torino, Italy. ${ }^{3}$ Cesky Metrologicky Institut (CMI), Okruzni 31 63800 Brno, Czech Republic. ${ }^{4}$ National Physical Laboratory (NPL), Hampton Road, Teddington, TW11 OLW, United Kingdom.

\section{Endnotes}

a We underline that for a general laser pulse period $\tau$, the formula for the $p_{\text {click }}$ becomes $p_{\text {click }}=\frac{q}{m * q+1}$ where $m$ is the integer part of the ratio $D / \tau$.

b The "click" rate of dark counts needs a correction due to effective dead time $D$ of the system. For a general observed "click" rate $\rho_{c, D}$ (in the presence of dead time $D$ ), the true "click" rate after dead time correction is: $\rho_{c}=\frac{\rho_{C, D}}{1-\rho_{C, D} D^{D}}$, that means: $\rho_{C, D}=\frac{\rho_{C}}{1+\rho_{C} D}$.

\section{Publisher's Note}

Springer Nature remains neutral with regard to jurisdictional claims in published maps and institutional affiliations.

Received: 14 July 2020 Accepted: 13 November 2020 Published online: 25 November 2020

\section{References}

1. Hadfield RH. Single-photon detectors for optical quantum information applications. Nat Photonics. 2009:3:696-705.

2. Stucki D, Ribordy G, Stefanov A, Zbinden H, Rarity JG, Wall T. Photon counting for quantum key distribution with peltier cooled InGaAs/InP APDs. J Mod Opt. 2001;48(13):1967-81.

3. Yuan ZL, Dixon AR, Dynes JF, Sharpe AW, Shields AJ. Practical gigahertz quantum key distribution based on avalanche photodiodes. New J Phys. 2009;11:1-11.

4. Gisin N, Ribordy G, Tittel W, Zbinden H. Quantum cryptography. Rev Mod Phys. 2002;74:145.

5. Lucamarini M, et al. Implementation Security of Quantum Cryptography. 2018. ETSI White Paper No. 27.

6. Feihu X, Xiongfeng M, Qiang Z, Hoi-Kwong L, Jian-Wei P. Secure quantum key distribution with realistic devices. arXiv:1903.09051 (2008).

7. Scarani V, Bechmann-Pasquinucci H, Cerf N, Dušek M, Lütkenhaus N, Peev M. The security of practical quantum key distribution. Rev Mod Phys. 2009;81:1301-48.

8. López M, Hofer H, Kück S. Detection efficiency calibration of single-photon silicon avalanche photodiodes traceable using double attenuator technique. J Mod Opt. 2015;62:S21-7. 
9. Lee HJ, Park S, Park HS, Hong KS, Lee D-H, Kim H, Cha M, Moon HS. Wavelength-scanning calibration of detection efficiency of single photon detectors by direct comparison with a photodiode. Metrologia. 2016;53(2):908.

10. Cheung JY, Chunnilall CJ, Porrovecchio G, Smid M, Theocharous E. Low optical power reference detector implemented in the validation of two independent techniques for calibrating photon-counting detectors. Opt Express. 2011;19(21):20347-63.

11. Chunnilall CJ, Lepert G, Allerton JJ, Hart CJ, Sinclair AG. Traceable metrology for characterizing quantum optical communication devices. Metrologia. 2014;51(6):S258-66.

12. Brida G, Castelletto S, Degiovanni IP, Genovese C, Novero C, Rastello ML. Towards an uncertainty budget in quantum-efficiency measurements with parametric fluorescence. Metrologia. 2000;37:629-32.

13. Migdall A, Castelletto S, Degiovanni IP, Rastello ML. Intercomparison of correlated-photon-based method to measure detector quantum efficiency. Appl Opt. 2002;41:2914-22.

14. Polyakov SV, Migdall AL. High accuracy verification of a correlated-photon-based method for determining photon-counting detection efficiency. Opt Express. 2007:15(4):1390-407.

15. Polyakov SV, Migdall AL. Quantum radiometry. J Mod Opt. 2009;56:1045-52.

16. Avella A, Brida G, Degiovanni IP, Genovese M, Gramegna M, Lolli L, Monticone E, Portesi C, Rajteri M, Rastello ML, Taralli E, Traina P, White M. Self consitent absolute calibration technique for photon number resolving detectors. Opt Express. 2011;19:23249-57.

17. Castelletto S, Degiovanni I, Rastello M. Evaluation of statistical noise in measurements based on correlated photons. J Opt Soc Am B. 2002;19(6):1247-58.

18. Müller I, Klein RM, Hollandt J, Ulm G. Traceable calibration of Si avalanche photodiodes using synchrotron radiation. Metrologia. 2012;49(2):152-5.

19. Gerrits T, Migdall A, Bienfang JC, Lehman JNSW, Splett J, Vayshenker I, Wang J. Calibration of free-space and fiber-coupled single-photon detectors. Metrologia. 2019;57:1-18.

20. Porrovecchio G, Šmid M, López M, Hofer H, Rodiek B, Kück S. Comparison at the sub-100 fW optical power level of calibrating a single-photon detector using a high-sensitive, low-noise silicon photodiode and the double attenuator technique. Metrologia. 2016:53:1115-22.

21. https://www.idquantique.com/single-photon-systems/products/. DI220 Infrared Single-Photon Detector.

22. Castelletto S, Degiovanni IP, Rastello ML. Theoretical aspects of photon number measurement. Metrologia. 2000:37:613-6.

23. Mountford J, Porrovecchio G, Smid M, Smid R. Development of a switched integrator amplifier for high-accuracy optical measurements. Appl Opt. 2008:47(31):5821-8.

24. Evaluation of measurement data - Guide to the expression of uncertainty in measurement, BIPM. 2008

25. Humer G, Peev M, Schaeff C, Ramelow S, Stipčević M, Ursin R. A simple and robust method for estimating afterpulsing in single photon detectors. J Lightwave Technol. 2015;33:3098-107.

26. Mueller I, Horansky RD, Lehman JH, Nam SW, Vayshenker I, Werner L, Wuebbeler G, White M. Verification of calibration methods for determining photon-counting detection efficiency using superconducting nano-wire single photon detectors. Opt Express. 2017;25(18):290285

27. Cox MG. The evaluation of key comparison data: an introduction. Metrologia. 2002;39:589-95.

28. Steele A, Douglas R. Extending En for measurement science. Metrologia. 2006;43:235-43.

29. Brida G, Castelletto S, Degiovanni IP, Novero C, Rastello ML. Quantum efficiency and dead time of single-photon counting photodiodes: a comparison between two measurement techniques. Metrologia. 2000;37:625-8.

\section{Submit your manuscript to a SpringerOpen ${ }^{\circ}$ journal and benefit from:}

- Convenient online submission

- Rigorous peer review

- Open access: articles freely available online

- High visibility within the field

- Retaining the copyright to your article

Submit your next manuscript at $\gg$ springeropen.com 